\title{
Urinary Tract Infection: Bacteriological Profile and Its Antibiotic Susceptibility Pattern in a Tertiary Care Hospital in Bangalore, India
}

\author{
P.R. Lyra, Vivek Hittinahalli* and K.T. Sangeetha \\ Department of Microbiology, East Point College of Medical Sciences and Research Centre, \\ Bangalore, Karnataka, India \\ *Corresponding author
}

\section{Keywords}

Enterobacteriaceae,

Piperacillin/

tazobactam,

Klebsiella spp.

\section{Article Info}

Accepted:

10 June 2019

Available Online:

10 July 2019

\section{A B S T R A C T}

Urinary tract infection (UTI) is one of the most common bacterial illnesses and hence one of the most important indications for antibiotic treatment. Current knowledge of the common organisms implicated in causing UTI in the local community and surveillance to monitor the changes in susceptibility of uropathogens are imperative to ensure appropriate therapy. The study objectives were to assess the proportion of UTI caused by each of the common urinary pathogens, to study the antibiotic drug sensitivity patterns by analyzing the culture and sensitivity reports and to identify the drugs which would be potentially favourable candidates for empirical therapy in the study locale. A total of 210 culture positive bacterial isolates from 514 urine samples; submitted over a period of 6 months were included in this study. Identification of bacterial isolates was done by standard biochemical profile of the organisms. The antimicrobial susceptibility of culture positive bacterial isolates was performed by disk diffusion method as recommended by Clinical Laboratory Standard Institute guidelines (CLSI). The predominant organism isolated was E. coli (51.5\%) followed by Klebsiella spp (25\%) and Pseudomonas spp (14\%). Among the 210 samples which yielded growth $150(71.4 \%)$ were from females and $60(28.6 \%)$ were from males. $160(76 \%)$ samples were from inpatients whereas $50(24 \%)$ samples were from outpatients. The susceptibility pattern of E. coli showed that $100 \%$ of the bacterial isolates were sensitive to imipenem and meropenem, $95 \%$ to piperacillin tazobactam and $92 \%$ to nitrofurantoin. Klebsiella spp. showed $100 \%$ sensitivity to imipenem and meropenem followed by piperacillin - tazobactam and amikacin. In conclusion majority of the bacterial isolates were sensitive to imipenem, meropenem, and piperacillin/tazobactam while susceptibility to cephalosporins was very low. Among the oral antimicrobials, nitrofurantoin showed good susceptibility against Enterobacteriaceae family.

\section{Introduction}

Urinary tract infections (UTIs) are one of the most common human bacterial infections both in the community and hospital setting. ${ }^{1-3}$ It is estimated that $20 \%$ or more of the female population suffers some form of UTI in their lifetime. Infection in the male population remains uncommon through the fifth decade of life, when enlargement of the prostate begins to interfere with emptying of the bladder. $^{4}$ 
Members of the family Enterobacteriaceae are a well-known cause of urinary tract infections. E. coli is reported to be the most common bacteria with prevalence as high as $71 \%$ and resistance to commonly used antimicrobials. ${ }^{5}$ Other Gram negative organisms like $P$. aeruginosa and Gram positive organisms like Enterococcus spp. are the common urinary tract bacteria frequently involved in hospital acquired infections. ${ }^{6}$

In spite of the availability and use of the antimicrobial drugs, UTIs caused by bacteria have been showing increasing trends. The extensive and inappropriate use of antimicrobial agents has invariably resulted in the development of antibiotic resistance which, in recent years, has become a major problem worldwide. ${ }^{7}$

In patients with suspected UTI, antibiotic treatment is usually started empirically, before urine culture results are available. To ensure appropriate treatment, knowledge of the organisms that cause UTI and their antibiotic susceptibility is mandatory. ${ }^{8}$

Hence this study was undertaken to determine the common pathogens responsible for UTI and their antibiotic sensitivity pattern.

\section{Materials and Methods}

The study was conducted in the Department Of Microbiology, East Point College of Medical Sciences \& Research Centre, Bangalore from August 2017 to January 2018. A total of 514 patients with clinically suspected urinary tract infection during August 2017 to January 2018 were included in the study.

\section{Collection of Urine Samples}

Mid-stream urine samples were collected using sterile, wide mouthed container with screw cap tops. On the urine sample bottles were indicated name, age, sex, and time of collection along with requisition forms. The samples were subjected to microscopy and culture.

\section{Sample processing}

\section{Microscopy}

The urine samples were mixed and aliquots centrifuged at $5000 \mathrm{rpm}$ for $5 \mathrm{~min}$. The deposits were examined using both 10X and $40 X$ objectives. Samples with $\geq 10$ white blood cells $/ \mathrm{mm}^{3}$ were regarded as pyuric. A volume of the urine samples were applied to a glass microscope slide, allowed to air dry, stained with gram stain, and examined microscopically.

\section{Culture}

A calibrated sterile micron wire loop for the semi-quantitative method was used for the plating and it has a $4.0 \mathrm{~mm}$ diameter designed to deliver $0.01 \mathrm{ml}$. A loopful of the well mixed urine sample was inoculated into duplicate plates of Blood and CLED agar. All plates were then incubated at $37^{\circ} \mathrm{C}$ aerobically for 24 $\mathrm{h}$. The plates were then examined for bacterial growth. Bacterial isolates were identified generally using biochemical reactions. The bacterial colonies were counted and multiplied by 100 to give an estimate of the number of bacteria present per milliliter of urine. A significant bacterial count was taken as any count equal to or in excess of $10,0000 \mathrm{cfu}$ $/ \mathrm{ml} .^{9}$ Antibacterial susceptibility of the isolates was done using Kirby-Bauer disk diffusion method following CLSI protocol ${ }^{10}$. Commercially available standard antibiotic discs (Himedia) were used. The zones of inhibition were measured and recorded according to the CLSI guidelines. The isolates were tested for Ampicillin (AMP) $(10 \mu \mathrm{g})$, Cefazolin (CZ) $(30 \mu \mathrm{g})$, Cefuroxime (CXM) 
$(30 \mu \mathrm{g})$, Ceftriaxone (CTR) $(30 \mu \mathrm{g})$, Cefepime (CPM) $(30 \mu \mathrm{g})$, Nitrofurantoin (NIT) $(300 \mu \mathrm{g})$, Amoxicillin-Clavulanic acid (AMC) (10/20 $\mu \mathrm{g})$, Co-trimoxazole (COT) $(1.25 / 23.75 \mu \mathrm{g})$, Ciprofloxacin(CIP) $(5 \mu \mathrm{g})$, Gentamicin (G) $(10 \mu \mathrm{g})$, Amikacin (AK) $(30 \mu \mathrm{g})$, Tobramycin (TOB) $(10 \mu \mathrm{g})$, Piperacillin-Tazobactam (PIT) $(100 / 10 \mu \mathrm{g})$ and Imipenem (I) $(10 \mu \mathrm{g})$, Meropenem (MR) (10 $\mu \mathrm{g})$ (Hi-media, Mumbai). S. aureus (ATCC 25923), E. coli (ATCC 25922) and $P$. aeruginosa (ATCC 27853) were used as control strains.

\section{Results and Discussion}

Out of the 514 urine samples received for culture and sensitivity during the study period, $210(41 \%)$ yielded significant bacteriuria. 150 (71.4\%) were from females and 60 (28.6\%) were from males.160 (76\%) samples were from inpatients whereas 50 (24\%) samples were from outpatients.

The predominant organism isolated was E.coli (51.5\%) followed by Klebsiella spp (25\%) and Pseudomonas spp (14\%).

Bacterial infections of the urinary tract are one of the frequent cause for seeking medical attention in community. ${ }^{11}$ Effective management of patients suffering from bacterial UTIs commonly relies on the identification of the bacterial isolate and the selection of an effective antibiotic agent used for the treatment of bacterial organisms in question. $^{12}$ Antimicrobial resistance is a serious public health threat.

Treatment failure is caused by resistance developed by different bacterial pathogens against commonly used antimicrobials.

In community and hospital settings the etiology of UTIs and the antimicrobial susceptibility of UTI causing bacteria's have been changing over the years. ${ }^{13,14}$
In this study, the bulk of the urinary isolates were from female patients as UTIs are frequent in females due to short urethra.

The most common urinary tract bacteria was found to be $E$. coli, a frequent causative agent of UTIs. A similar study conducted at Department of Microbiology, Armed Forces Institute of Pathology, Rawalpindi in 2010 and at Mayo Hospital, Lahore in 2013 revealed $E$. coli as the most common bacteria accounting for $63 \%$ and $80 \%$ of the total culture positive isolates. ${ }^{15,16}$ A similar study conducted in Peshawar, Khyber Pakhtunkhwa Pakistan has revealed similar results showing E. coli $(77 \%)$ as the predominant uropathogen. ${ }^{17}$ The frequency of $E$. coli as the causative agent of UTIs was found to be $80-90 \%$ in two similar studies carried out in Canada and Ethiopia in the recent years. ${ }^{18,19}$

The second most common urinary isolate in this study was Klebsiella spp which is similar to other studies where the second commonest reported isolates were Staphylococcus spp. and K. pneumoniae ${ }^{20,21,22}$ (Table 1).

Pseudomonas aeruginosa was the third most common isolate in our study. A study conducted at Department of Microbiology, Armed Forces Institute of Pathology, Rawalpindi in 2012 revealed Pseudomonas aeruginosa as the second most common isolate in UTI. ${ }^{23}$

In this study, $100 \%$ of $E$. coli isolates were susceptible to imipenem, the result being consistent with similar study carried out at AFIP, two years ago and the study conducted in Peshawar. ${ }^{15,}{ }^{17}$. These results are also similar to earlier studies carried out in India where $96 \%$ of E. coli isolates were susceptible to imipenem. ${ }^{24}$.The results of this study are contrary to a similar study conducted recently in Lahore, Pakistan, where E. coli showed $44 \%$ resistance to carbapenems ${ }^{16}$ (Fig. 1-3). 
Fig.1 Antibiotic sensitivity pattern of E. coli (\%)

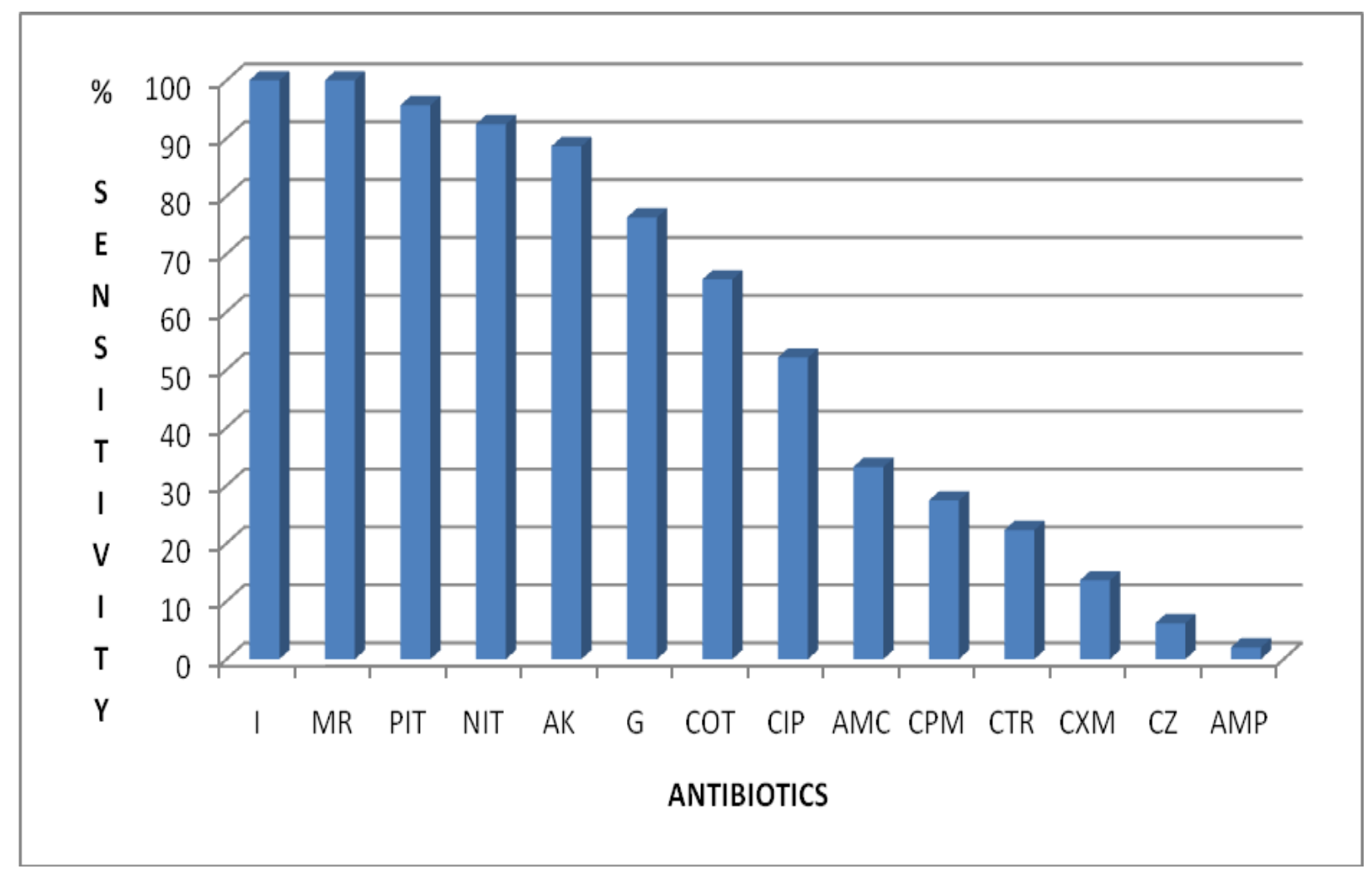

Fig.2 Antibiotic sensitivity pattern of Klebsiella spp.

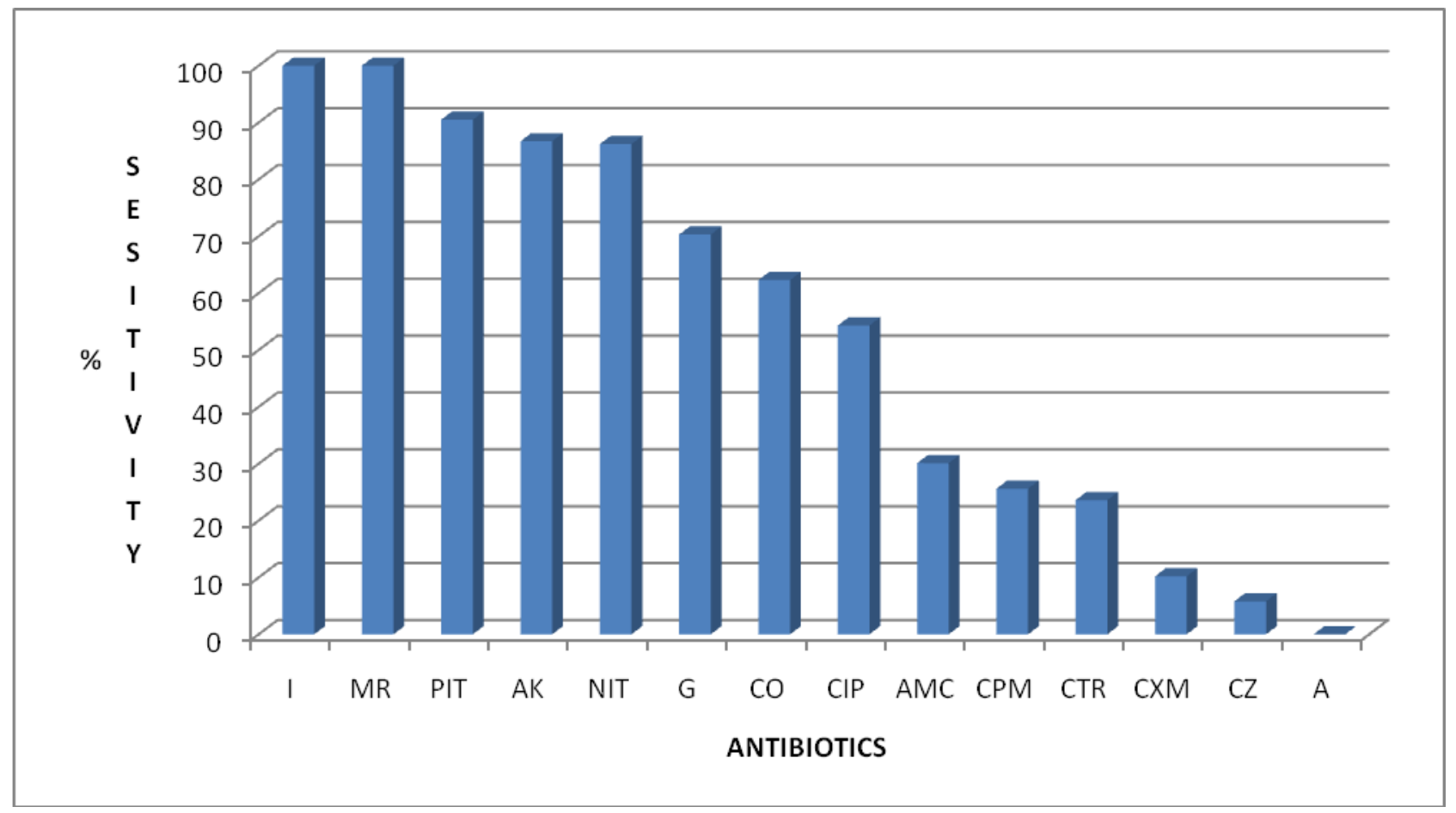


Fig.3 Antibiotic sensitivity pattern of Pseudomonas spp.

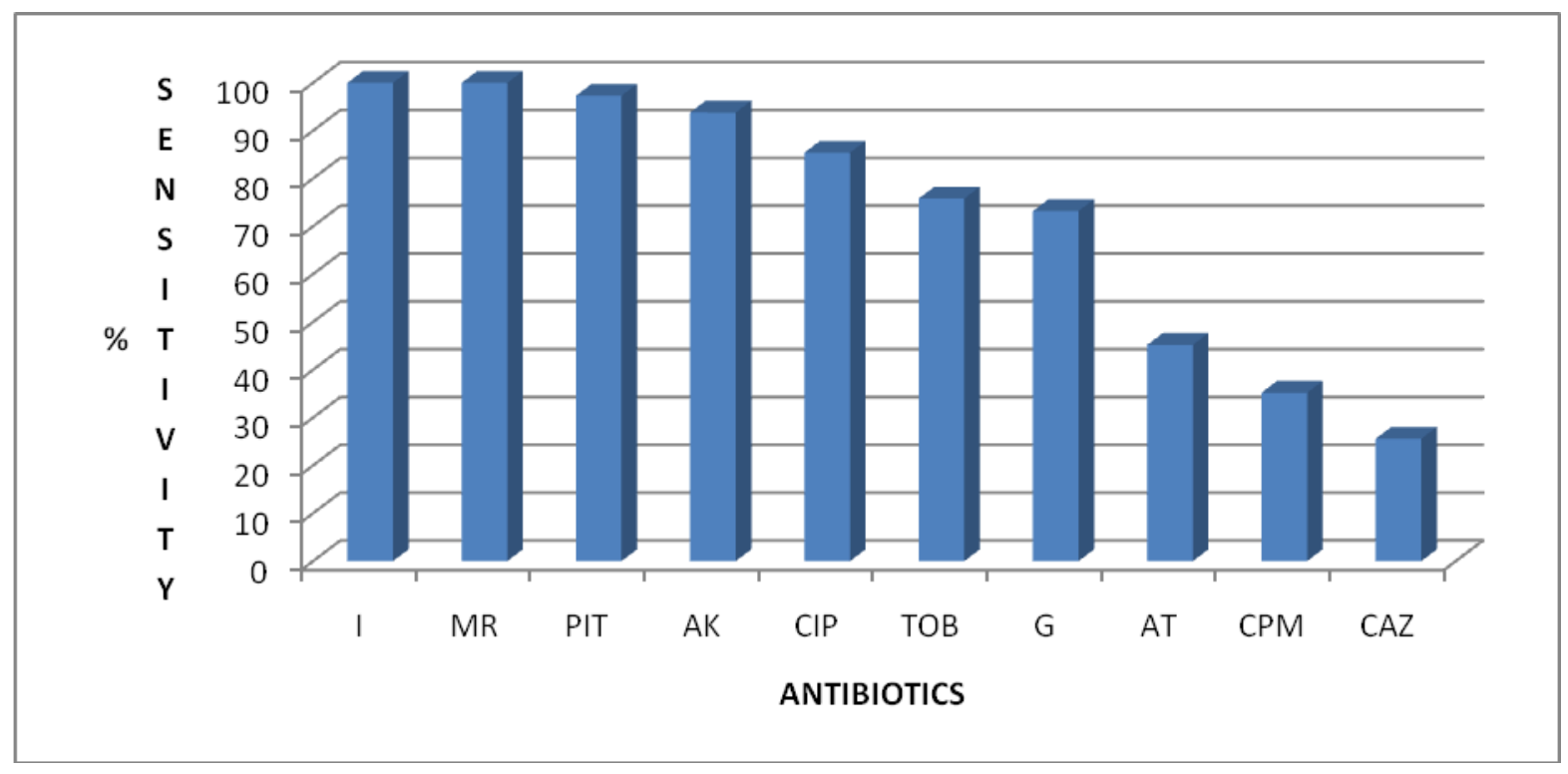

Table.1 Distribution of bacterial pathogens isolated from urine samples

\begin{tabular}{|l|l|}
\hline Organism & No.of isolates (\%) \\
\hline E.coli & $109(51.5 \%)$ \\
\hline Klebsiella spp & $52(25 \%)$ \\
\hline Pseudomonas spp & $29(14 \%)$ \\
\hline Enterococcus spp & $6(3 \%)$ \\
\hline Staphylococcus aureus & $5(2 \%)$ \\
\hline Proteus spp & $4(2 \%)$ \\
\hline Citrobacter spp & $2(1 \%)$ \\
\hline Acinetobacter spp & $2(1 \%)$ \\
\hline Serratia spp & $1(0.5 \%)$ \\
\hline Total & 210 \\
\hline
\end{tabular}

In this study, the susceptibility of E. coli to Nitrofurantoin was $92 \%$ compared to $94 \%$ in a study done in London. ${ }^{25}$. Nitrofurantoin is effective against many Gram positive and Gram negative urinary isolates and activity of this antimicrobial is greatly enhanced at $\mathrm{pH}$ 5.5 and below. It is a cheap antimicrobial and can be given orally for months for the suppression of chronic UTIs. It shows that Nitrofurantoin is still effective against majority of the urinary isolates and can be used prophylactically for recurrent urinary tract infections. ${ }^{23}$
Similarly, another important oral antimicrobial used for empirical treatment of uncomplicated UTIs in our setup is trimethoprim/sulfamethoxazole. The susceptibility of $E$. coli to cotrimoxazole was found to be $65 \%$ in this study. In countries where resistance is low $(<20 \%)$, trimethoprim-sulfamethoxazole can still be a valid first-line antibiotic, and in many countries where its use has been limited for years, in consideration of the evidence-based international guidelines, the resistance rate has receded and made possible the 
reintroduction of this combination as a therapeutic strategy. ${ }^{26}$

As far as the antimicrobial sensitivity of quinolones to $E$. coli is concerned, the susceptibility to ciprofloxacin was $52 \%$. The situation is quite different with $E$. coli isolated in a study carried out in London where $94 \%$ of bacterial isolates were susceptible to ciprofloxacin. ${ }^{25}$.These contrasting results clearly suggest the injudicious use of quinolones in this part of the world has led to deteriorating susceptibility to this important antimicrobial group.

In this study, Klebsiella spp. isolates showed $100 \%$ susceptibility to Imipenem and Meropenem followed by Piperacillin Tazobactam and Amikacin. The antimicrobial susceptibility of Enterobacteriaceae has revealed that imipenem was the most effective antibiotic similar to other studies. In vitro activity of oral antimicrobials such as fluroquinolones and cephalosporins was low comparable to other studies performed in Pakistan, which is a worrying trend as far as the oral antibiotics are concerned. ${ }^{17}$

Carbapenems, Amikacin, Ceftazidime and antipseudomonal penicillin such as Piperacillin are the recommended antibiotics to treat UTIs caused by $P$. aeruginosa. The antimicrobial susceptibility profile of Pseudomonas aeruginosa in this study revealed that $100 \%$ of the isolates were sensitive to Imipenem \& Meropenem followed by Piperacillin - Tazobactam and Amikacin. Only very few isolates were sensitive to Ceftazidime and Cefepime. This is a worrying trend with an indication that $P$. aeruginosa is gradually developing resistance against antipseudomonal cephalosporins.

The increasing rates of resistance to uropathogenic isolates warrant evaluation of other antimicrobials such as fosfomycin which can safely be given orally and is highly effective against many uropathogens. ${ }^{27}$ The results of this study will benefit clinicians to know the local pattern of antimicrobial susceptibilities and formulate the empirical antibiotic strategies in patients presenting with UTIs.

E. coli was the predominant pathogen causing UTIs in our population followed by Pseudomonas spp. A majority of the isolates were sensitive to Imipenem and Piperacillin/Tazobactam limiting the use of oral antimicrobials commonly used to treat UTIs. As far as the oral antibiotics are concerned, Nitrofurantoin revealed encouraging results proving to be the effective oral antibiotic in this study. As drug resistance among bacterial pathogens is an evolving process, routine surveillance and monitoring studies should be conducted in different parts of the country to provide physicians, an effective knowledge regarding the empirical treatment of UTIs in that particular area.

\section{References}

1. Akortha EE, Ibadin OK. Incidence and antibiotic susceptibility pattern of Staphylococcus aureus amongst patients with urinary tract infection in UBTH Benin City, Nigeria. Afr J Biotechnol 2008; 7:1637-40.

2. Dalela G, Gupta S, Jain DK, Mehta P. Antibiotic resistance pattern in uropathogens at a tertiary care hospital at Jhalawar with special reference to ESBL, Amp-C $\square$-Lactamase and MRSA production. J Clin Diagn Res 2012; 6:645-51.

3. Bhattacharya S. ESBL- From petri dish to the patient. Indian J Med Microbiol 2006; 24:20-4. 
4. Salek, SB., 1992. Infective syndrome in medical microbiology,4th edition, pp. 740

5. Thabet L, Messadi AA, Meddeb B, Mbarek A, Turki A, Ben RS. Bacteriological profile of urinary tract infection in women in Aziza Othmana Hospital: a 495 cases study. La Tunisie Medicale 2010; 88:898-901.

6. Minardi D, d'Anzeo G, Cantoro D, Conti A, Muzzonigro G. Urinary tract infections in women: etiology and treatment options. Int J Gen Med 2011; 4:333-43.

7. Enayat K, Fariba F, Bahram N. Asymptomatic bacteriuria among pregnant women referred to outpatient clinics in Sanandaj, Iran. Int Braz J Urol 2008; 34:699-707.

8. Rock W, Colodner R, Chazan B, Elias M, Raz R. Ten years surveillance of antimicrobial susceptibility of community Acquired Escherichia coli and other uropathogens in Northern Israel. Israel Med Assoc J 2007; 803-5.

9. Collee JG, Fraser AG, Marmion BP, Simmons A, Mackie and McCartney. Practical Medical Microbiology. $14^{\text {th }}$ edition.Churchill Living stone 1996; 413-414.

10. Clinical and Laboratory Standard Institute (CLSI). Performance standard for antimicrobial susceptibility testing: supplement M100-, $29^{\text {th }}$ edition. Wayne, PA: CLSI; 2019.

11. Kebira AN, Ochola P, Khamadi SA. Isolation and antimicrobial susceptibility testing of Escherichia coli causing urinary tract infections. $J$ Appl Biosci 2009; 22:1320-25.

12. Water G, Harrison B, Kunin G. Urinary tract infection. N Engl J Med 1996; 24850.

13. New HC. Urinary tract infections. Am J Med. 1996; 100(Suppl.4A): S63-70.
14. Jones RN. Impact of changing pathogens and antimicrobial susceptibility pattern in treatment of serious infections in hospitalized patients. Am J Med. 1996; 100 (Suppl.6A): S3-12.

15. Amjad A, Mirza IA, Abbasi SA, Farwa U, Sattar A, Qureshi ZA. Spectrum and antimicrobial susceptibility pattern of pathogens causing urinary tract infection: experience in a tertiary care setting. Infect Dis J 2011; 20:297-301.

16. Sabir S, Anjum AA, Ijaz T, Ali MA, Khan MR, Nawaz M. Isolation and antibiotic susceptibility of $E$. coli from urinary tract infections in a tertiary care hospital. Pak J Med Sci 2014; 30:

17. Shahzad KA, Ullah F, Muhammad K, Khatoon F, Qazi MH, Ahmed I. Multiple drug resistance patterns in urinary tract infection patients in Peshawar, Khyber Pukhtunkhwa (KPK) Pakistan. J Inf Mol Biol 2013; 1:67-70.

18. Ronald A. The etiology of urinary tract infection: traditional and emerging pathogens. Am J Med 2002; 113:14-9.

19. Tessema B, Kassu A, Mulu A, Yismaw G. Predominant isolates of urinary tract pathogens and their susceptibility patterns in Gonder Univesity Teaching Hospital North west Ethiopia. Ethio Med $J$ 2007; 45:61-7.

20. Sabir S, Anjum AA, Ijaz T, Ali MA, Khan MR, Nawaz M. Isolation and antibiotic susceptibility of $E$. coli from urinary tract infections in a tertiary care hospital. Pak J Med Sci 2014; 30:389.

21. Shahzad KA, Ullah F, Muhammad K, Khatoon F, Qazi MH, Ahmed I. Multiple drug resistance patterns in urinary tract infection patients in Peshawar, Khyber Pukhtunkhwa (KPK) Pakistan. J Inf Mol Biol 2013; 1:67-70.

22. Chin BS, Kim MS, Han SH. Risk factors of all cause in-hospital mortality among Korean elderly bacteremic 
urinary tract infection (UTI) patients. Arch Gerontol Geriatr 2011; 52:50-5.

23. Inam Ullah Khan, Irfan Ali Mirza, Aamer Ikram, Amna Afzal, Shamshad Ali, Aamir Hussain, Muhammad Fayyaz and Tahir Ghafoor. Antimicrobial Susceptibility Pattern of Bacteria Isolated from Patients with Urinary Tract Infection. Journal of the College of Physicians and Surgeons Pakistan 2014, Vol. 24 (11): 840-844.

24. Eshwarappa M, Dosegowda R, Aprameya IV, Khan MW, Kumar PS, Kempegowda. Clinico-microbiological profile of urinary tract infection in south India. Indian J Nephrol 2011; 21:30-36.

25. Bean DC, Krahe D, Wareham DW. Antimicrobial resistance in community and nosocomial isolates of Escherichia coli urinary tract isolates, London 20052006. Ann Clin Microbiol Antimicrob 2008; 7:13.

26. Minardi D, d'Anzeo G, Cantoro D, Conti A, Muzzonigro G. Urinary tract infections in women: etiology and treatment options. Int J Gen Med 2011; 4:333-43.

27. Pullukcu H, Tasbakan M, Sipahi OR, Yamazhan T, Aydemir S, Ulusoy S. Fosfomycin in the treatment of extended spectrum b-lactamase producing Escherichia coli related lower urinary tract infection. Int $J$ Antimicrob Agents 2007; 29:62-5.

\section{How to cite this article:}

Lyra, P.R., Vivek Hittinahalli and Sangeetha, K.T. 2019. Urinary Tract Infection: Bacteriological Profile and Its Antibiotic Susceptibility Pattern in a Tertiary Care Hospital in Bangalore, India. Int.J.Curr.Microbiol.App.Sci. 8(07): 853-860. doi: https://doi.org/10.20546/ijcmas.2019.807.102 